\title{
Una imagen atípica para un tumor típico
}

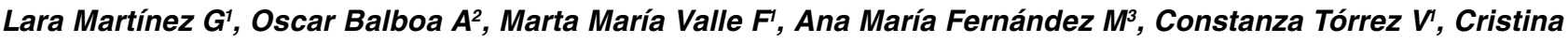
Antolín P3.

1. Médico residente de radiodiagnóstico, Complejo Asistencial de León. León - España.

2. Médico adjunto del servicio de radiodiagnóstico, Complejo Hospitalario Universitario de Santiago de Compostela. Santiago de Compostela - España.

3. Médico adjunto del servicio de radiodiagnóstico, Complejo Asistencial de León. León - España.

\section{An atypical imaging for the most typical intracranial tumor}

Abstract. Meningiomas are the most common benign intracranial tumors in adults, accounting for $20 \%$ of all brain tumors. Only 1-4\% have cystic changes. The relationship between the cystic and solid component of mixed meningiomas was used by several authors to create up to four different classifications of this subtype of meningiomas. CT diagnosis is challenging in this subtype of meningiomas because of the difficulty of distinguishing between different intracranial lesions that also have associated cystic component. We present the case of a 49-year-old male patient with episodes of amaurosis fugax. On CT, a solid lesion was identified in the left frontotemporal region, with a large cystic component that produced an important mass effect and midline displacement. A study was completed by MR and arteriography and finally was diagnosed as mixed meningioma, which after tumor embolization via the middle meningeal artery, was surgically resected.

Keyword: Meningioma, atypical, cystic, mixed, intracranial.

Resumen. Los meningiomas son los tumores intracraneales benignos más frecuentes en adultos, suponen el $20 \%$ de todos los tumores cerebrales. Solamente el 1-4\% presentan cambios quísticos. La relación entre el componente quístico y sólido de los meningiomas mixtos fue utilizada por varios autores para crear hasta cuatro clasificaciones diferentes de este subtipo de meningiomas. El diagnóstico mediante TC resulta muy complejo ante este subtipo de meningiomas por la dificultad de discernir entre diferentes lesiones intracraneales que también presentan componente quístico asociado. Presentamos el caso de un paciente varón de 49 años que presenta episodios de amaurosis fugax. En la TC se identificó una lesión sólida en localización frontotemporal izquierda, con gran componente quístico que producía importante efecto de masa y desplazaba la línea media. Se completó estudio mediante RM y arteriografía y finalmente fue diagnosticado como meningioma mixto, que tras embolización tumoral desde arteria meníngea media, se resecó quirúrgicamente.

Palabras clave: Meningioma, atípico, quístico, mixto, intracraneal.

Martínez L, et al. Una imagen atípica para un tumor típico. Rev Chil Radiol 2018; 24(2): 79-83.

Correspondencia: larissa233@hotmail.com

Trabajo enviado el 30 de octubre de 2017. Aceptado para publicación el 23 de mayo de 2018.

\section{Presentación del caso clínico}

Paciente de sexo masculino de 49 años que acude a urgencias por cuadro de 4 meses de evolución caracterizado por alteraciones visuales tipo amaurosis fugax sobre ojo izquierdo que se acompañan, en las últimas dos semanas, de cuadros de mareo inespecífico y un TEC secundario al mareo sin repercusión neurológica. La evaluación con fondo de ojo en urgencias demuestra edema de papila izquierda motivo por el cual se solicita un TC cerebral.

En los antecedentes personales del paciente: ExADVP, Hepatitis C (Desconocemos genotipo y carga viral). A tratamiento actual con metadona.

En la exploración neurológica destaca una paresia facial central.

Pruebas complementarias (Figuras 1, 2, 3, 4, 5, $6,7$ y 8$)$. 


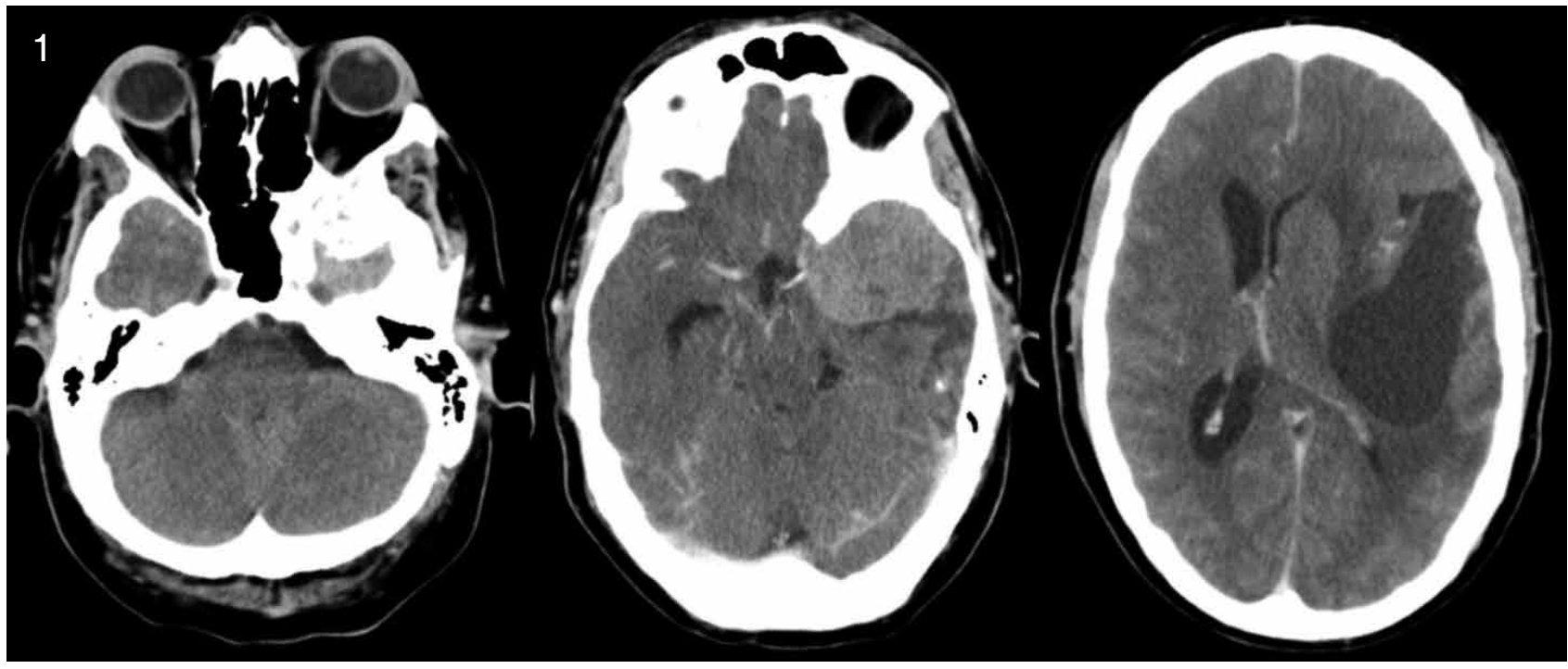

Figura 1. TC secuencial de cráneo con contraste: Esclerosis del ala mayor del esfenoides izquierdo. Lesión redondeada e isodensa temporal izquierda y edema digitiforme asociado. Lesión isodensa temporal izquierda e importante edema que produce efecto de masa y desplaza la línea media acompañado de gran componente quístico craneal a la lesión sólida. Tras la administración de civ la lesión temporal capta contraste de manera homogénea.
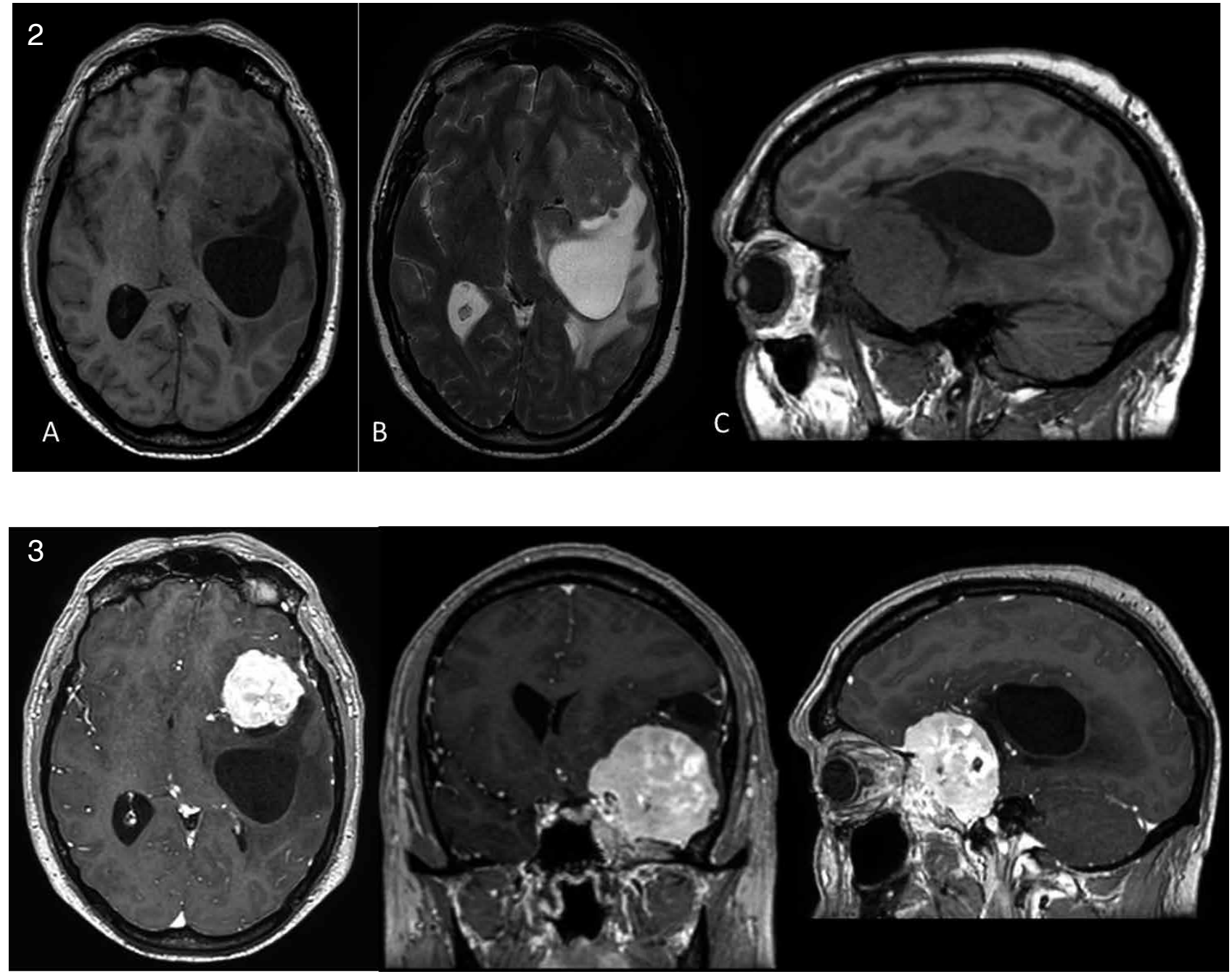


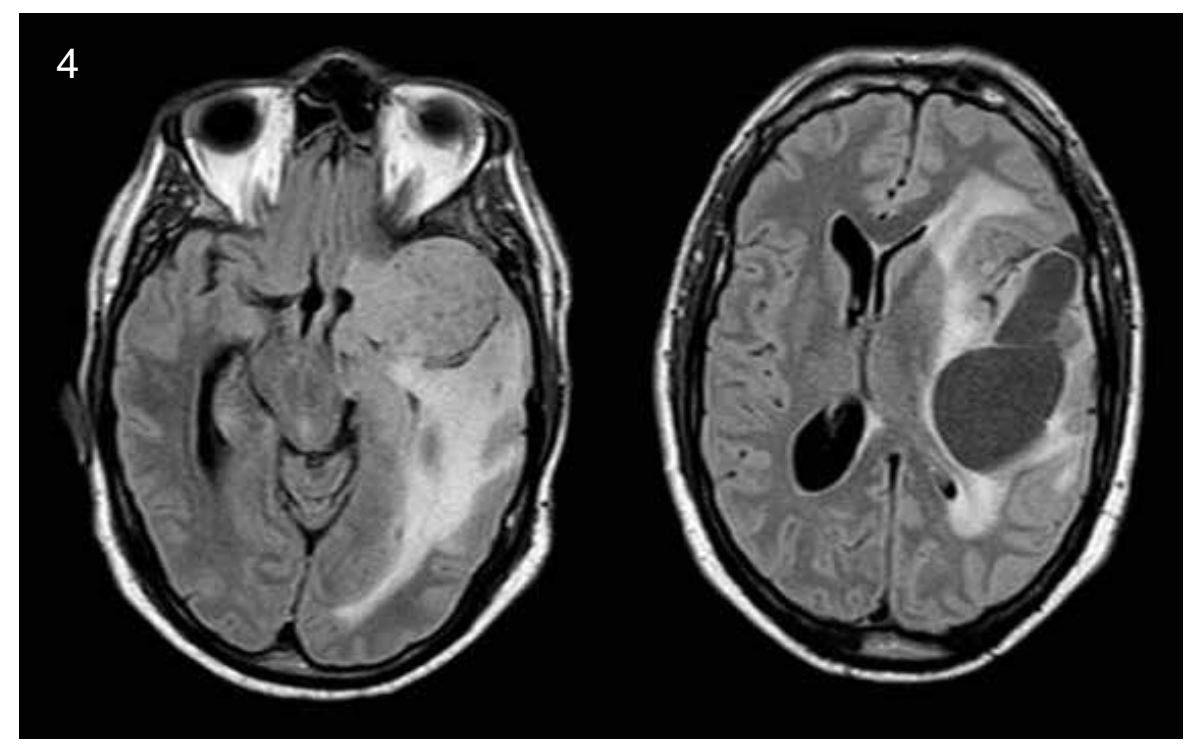

Figuras 2, 3, 4. RM Cerebro: (2) A: Secuencia ponderada en T1 axial: Lesión temporo-frontal izquierda regularmente definida e hipointensa respecto a la sustancia blanca. B: Secuencia ponderada en TA axial: Importante componente quístico asociada a la lesión previamente descrita y presencia de edema perilesional que condiciona efecto de masa sobre los surcos corticales adyacentes, colapso casi completo del VL homolateral y desviación de la línea media hacia la derecha. C: Secuencia ponderada en T1 sagital: Lesión temporo-frontal izquierda hipointensa respecto a la sustancia blanca y asociada a edema perilesional. (3) Secuencias ponderadas en T1 tras la administración de Gadolinio en los planos axial, coronal y sagital: Realce intenso de la lesión sólida sin realce del componente quístico. (4) Adquisiciones axiales en secuencias FLAIR-T2: Importante edema asociado a la lesión sólida que condiciona efecto de masa sobre los surcos corticales adyacentes.
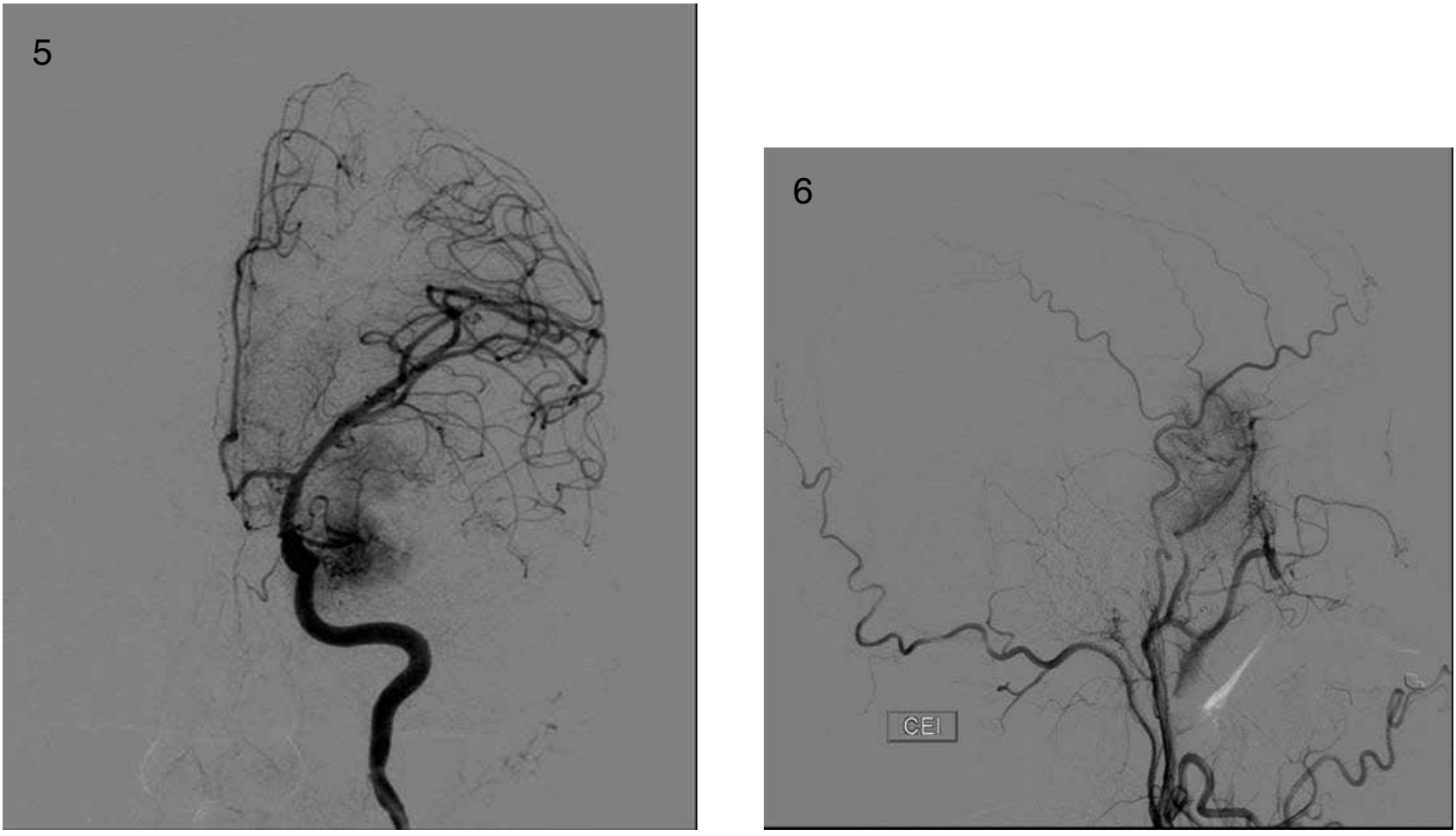

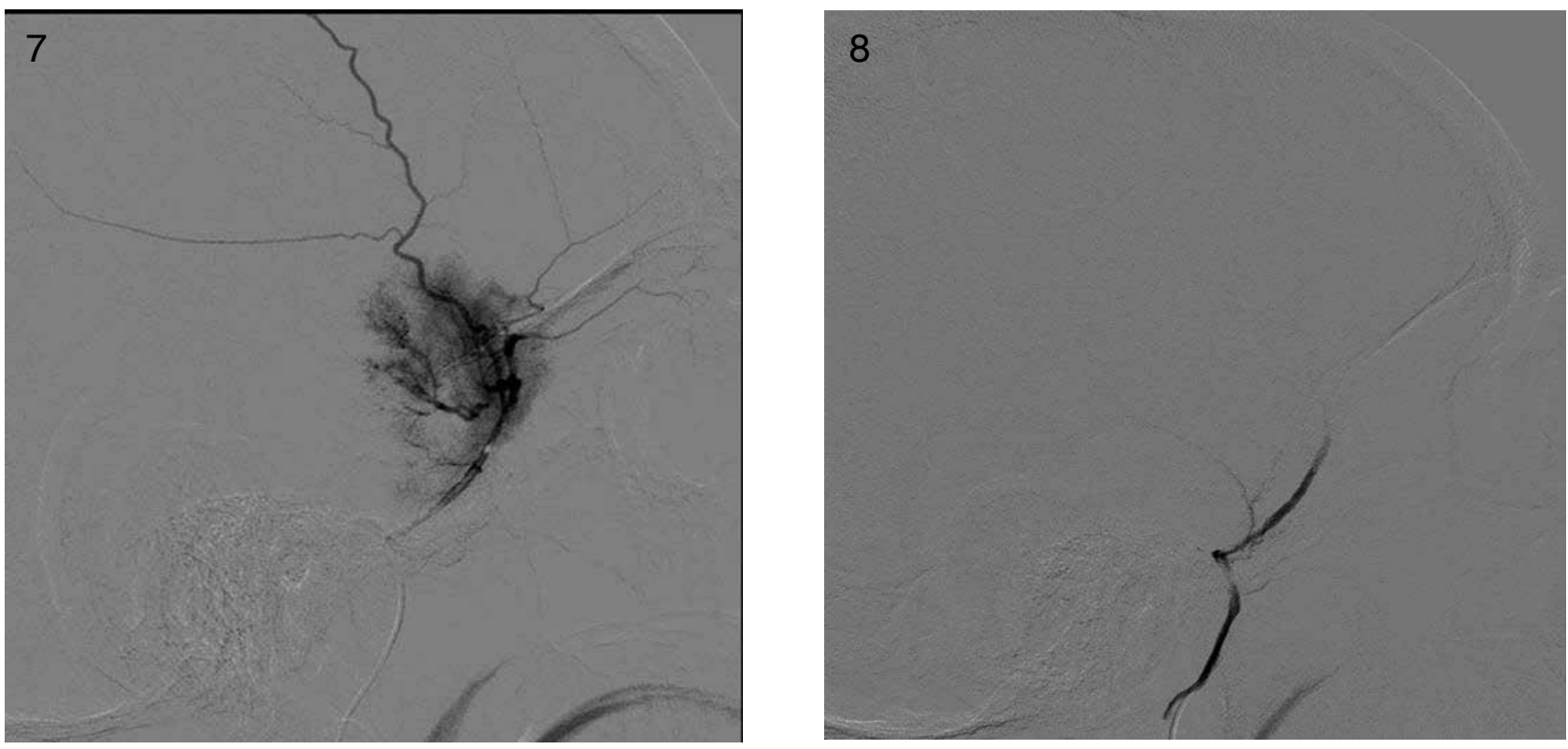

Figuras 5, 6, 7, 8. Arteriografía: (5) Arteriografía carótida interna izquierda donde se identifica teñido tumoral desde arterias meníngeas de la carótida y desplazamiento superior de la arteria cerebral media izquierda. (6) Arteriografía carótida externa izquierda donde se identifica teñido tumoral desde las ramas de la arteria meníngea media. (7) Arteriografía selectiva de la meníngea media donde claramente se objetiva un gran componente de teñido tumoral. (8) Arteriografía selectiva desde la meníngea media izquierda una vez embolizadas las aferencias tumorales.

\section{Diagnóstico anatomo-patológico tras resección quirúrgica previa embolización arterial \\ Meningioma meningotelial y Psamomatoso.}

\section{Discusión}

Los meningiomas son el tumor primario intracraneal más común (14-20\%, según las series) y predominan en las mujeres (2:1). La mayoría de los casos presentan características morfológicas típicas: masa extra-axial con marcado y homogéneo realce tras la administración de contraste. Existen algunos subtipos que convierten en un desafío su identificación por la imagen en TC ${ }^{1}$. Los meningiomas con componente quístico son uno de estos subtipos que plantean un diagnóstico diferencial con otras lesiones intracraneales que también presentan componente quístico asociado. En estos casos, la utilización de otras pruebas de imagen como la RM, la arteriografía cerebral y el estudio histopatológico son fundamentales para conseguir un correcto diagnóstico².

Estos meningiomas con componente quístico asociado son poco frecuentes (1-4\% de los casos). La incidencia es más alta en la edad pediátrica y su localización más frecuente es en la convexidad cerebral y, en segundo lugar, a nivel parafacinos ${ }^{3}$.

Rengachary describe dos clases de quistes: los intratumorales y los peritumorales; Los primeros son raros y los segundos son de gran tamaño, generalmente uniloculares y contienen fluido xantocrómico con alto contenido proteico ${ }^{4}$. La etiopatogenia de los cambios quísticos observados radiológicamente en los meningiomas no está clara; se han postulado algunos mecanismos como cambios degenerativos, secretorios, gliosis reactiva, necrosis, isquémica o hemorragia intratumoral y desmielinización causada por el edema en la sustancia blanca y déficit de perfusión tisular, en los casos con componente quístico intratumoral, y mecanismos como el atrapamiento y loculación del líquido cefalorraquídeo, secreción de células tumorales, quiste aracnoideo o degeneración quística periférica, en los casos de componente quístico peritumoral ${ }^{4,5}$. En estos casos, se ha llegado a postular que el quiste es el resultado de una respuesta glial a la presencia del meningioma o como una etapa final del edema peritumoral secundario a cambios hidrodinámicos, desmielinización o hemorragias ${ }^{6}$.

Aparte de la clasificación de Rengachary, existen varias clasificaciones de los meningiomas con componente quístico y todas ellas se basan en la relación del componente quístico con respecto al sólido y con la ubicación de dichos componentes, así Nauta et al. los clasificaron en 4 tipos:

- Nauta tipo 1: Componente quístico intratumoral y ubicación central.

- Nauta tipo 2: Componente quístico intratumoral y ubicación periférica.

- Nauta tipo 3: Componente quístico peritumoral y en el parénquima adyacente.

- Nauta tipo 4: Componente quístico entre el tumor y el tejido adyacente. 
Zee et al. sugirieron otro tipo de clasificación en 3 tipos dependiendo de la morfología, lo cual resulta más fácil a la hora de correlacionar con los hallazgos de la imagen ${ }^{10}$ :

- Tipo 1: Quistes intratumorales.

- Tipo 2: Quistes periféricos con pared formada por células tumorales.

- Tipo 3: Quistes periféricos en contacto con parénquima cerebral y con pared sin presencia de células tumorales.

Por otro lado, El-Fiki los clasifica según la relación entre la localización del quiste con su contenido ${ }^{3,5}$ :

- Tipo A: El contenido del quiste es similar al LCR y de ubicación extratumoral en el $55 \%$ de los casos. Escaso edema asociado (A1 o A2 dependiendo de si existe o no una membrana aracnoidea que separa el quiste del tumor).

- Tipo B: Contenido xantocrómico y edema moderado. Sin pared definida, B1, con pared definida, B2.

- Tipo C: El Quiste es intratumoral y con marcado edema periférico.

- Tipo D: Quistes mixtos.

La clínica acompañante va a depender de múltiples factores siendo los más importantes el tamaño de las lesiones, el edema asociado, la localización, la velocidad de crecimiento de la lesión ${ }^{5}$.

La mayoría presentan un comportamiento benigno, aunque, dependiendo de las series consultadas, del $8 \%$ al $20 \%$ de casos presentan un comportamiento agresivo o incluso maligno ${ }^{7,8}$.

En cuanto a la imagen, el tamaño del componente quístico es variable, pudiendo encontrar desde pequeñas lesiones sólidas con gran componente quístico que producen un importante efecto de masa y desplazan la línea media, lo que conlleva una sintomatología neurológica más grave, hasta incluso lesiones sólidas tan pequeñas que no llegan a ser identificables en la imagen. El diagnóstico de este tipo de tumores mediante TC es un reto ya que solo se diagnostican un $56 \%$ con esta técnica de imagen ${ }^{9,10}$. El diagnóstico diferencial, por la imagen radiológica, debe realizarse con tumores de estirpe glial (glioblastoma, oligoden- droglioma, etc), metástasis, linfomas y lesiones no neoplásicas como cisticercosis, absceso cerebral y quistes aracnoideos, entre otros ${ }^{10,11}$.

\section{Conclusión}

En nuestro caso y tras lo expuesto anteriormente, el paciente presenta un meningioma con componente quístico peritumoral, según Rengachary, Tipo 4, según la clasificación de Nauta, y tipo 3 de Zee.

\section{Referencias}

1. "Uncommon cranial meningioma: Key imaging features on conventional and advanced imaging". Zakhari N, Torres C, Castillo M, Nguyen TB. Clin Neuroradiol DOI 10.1007/s00062-017-0583-y.

2. "Cystic meningioma masquerading as a metastatic tumor: A case report". Ramanathan N, Kamaruddin KA, Othman A, Mustafa F, Awang MS. Malays J Med Sci. May-Jun 2016; 23(3): 92-94.

3. "Variante poco frecuente de meningiomas: Meningiomas quísticos". Rinaldi M, Mezzano E, Berra M, Olocco R, Pares H, Papalini F. Revista Argentina de Neurocirugía 2008; 22: 107.

4. "Meningioma quístico: Reporte de caso y revisión de literatura". Moscote LR, Sabogal Barrios R, Sabogal R. Revista ecuatoriana de Neurología 2007; 16(3): 219-221.

5. "Meningioma quístico". Elizondo Barriel LM, Gil Cruz JJ, Junco Martín RA, Ríos Castillo MC, Nuevas Matos J. Arch Neurocien (Mex) 2009; 14(3): 202-205.

6. "Cystic lesions associated with meningiomas". Maiuri F, Benvenuti D, De Simeone MR, Cirillo S, Corriero G, Giamundo A. Surg Neurol 1986; 26: 591-597.

7. "Meningiomas: unusual forms of intracranial neoplasms". Mena I, Noboa CA, Leone-Stay G, Vasconez JV, Cardenas-Mena B. Rev Neeurol 1998; 27: 50-52.

8. "Cystic lesions associated with intracraneal meningiomas". Rengachary S, Bamitzky S, Kepes JJ, Morants RA. Neurosurgery 1979; 4: 07.

9. "Cystic meningioma”. Liu M, Liu Y, Li X, Zhu S, Wu C. J Clin Neurosci. 2007; 14(9): 856-859.

10. "Magnetic resonance imaging of cystic meningiomas and its surgical implications". Zee CS, Chen T, Hinton DR, Tan M, Segall HD, Apuzzo ML. Neurosurgery. 1995; 36: 482-488.

11. "Cystic Meningioma". El-Fiki M, El-Henawy Y, AbdelArman N. Acta Neurochir (Wien) 1996; 138: 811-817.. 